\title{
The Analysis of an SIRS Epidemic Model with Discrete Delay on Scale-Free Network
}

\author{
Tao Li1 ${ }^{1}$,iming Liu' ${ }^{1}$, Baochen Li ${ }^{2}$ \\ ${ }^{1}$ Institute of Applied Mathematics, Shijiazhuang Mechanical Engineering College, Shijiazhuang, China \\ ${ }^{2}$ Department of Science and Research, Shijiazhuang Mechanical Engineering College, Shijiazhuang, China \\ Email: leo 119@163.com, lammath@163.com, testability83@163.com
}

Received 12 September 2015; accepted 26 October 2015; published 29 October 2015

Copyright (C) 2015 by authors and Scientific Research Publishing Inc.

This work is licensed under the Creative Commons Attribution International License (CC BY). http://creativecommons.org/licenses/by/4.0/

c) (i) Open Access

\section{Abstract}

A new epidemic SIRS model with discrete delay on scale-free network is presented. We give the formula of the basic reproductive number for the model and prove that the disease dies out when the basic reproductive number is less than unity, while the disease is uniformly persistent when the basic reproductive number is more than unity. Numerical simulations are given to demonstrate the main results.

\section{Keywords}

Scale-Free Network, Epidemic Spreading, Attractivity, Uniformly Persistence, Time Delay

\section{Introduction}

Since the modelling of the seminal works on the scale-free network, in which the probability of $p(k)$ for any node with $k$ links to other nodes is distributed according to the power law $p(k)=C k^{-\gamma}(2<\gamma \leq 3)$, suggested by Barabá and Albert [1], it is well known that the real disease transmission networks exhibit scale-free properties (see for example [2] [3]), and the spreading of epidemic disease on scale-free network has been studied by many researchers [4]-[21].

Continuous time deterministic epidemic models are traditionally formulated as systems of ordinary differential equations. More realistic models should include some peat states of these systems, and ideally, a real system should be modeled by delay differential equation. Time delay plays an important role in propagation process of the epidemic, we can simulate the latent period of infectious diseases, the infections period of patients and the immunity period of recovery of the disease with time delay. Much attention has been given to the dynamical behaviors of the epidemic spreading model with time delay on homogeneous network [18]. However, up to now, 
compared with studies of the dynamical behaviors of the epidemic models with time delays on hetergeneous network, only a few attentions have been paid to them on heterogeneous networks. Recently, Liu and Xu presented a delay differential equation SEIRS epidemic model with discrete time delays which represent the latent period and the immune period [19]. Liu and Deng et al. discussed epidemic SIS model with discrete time delay which represents the infectious period [20], they obtained the basic reproduction number and discussed the persistence of the disease. Wang and Wang et al. discussed an epidemic SIR model with discrete time delay which represented the latent period [21]. Motivated by these, in this paper, we will present a suitable epidemic SIRS model with discrete delay which represents the infectious period on scale-free network using functional differential equations to investigate the epidemic spreading.

The rest of this paper is organized as follows: The SIRS model on scale-free network with discrete delay is presented in Section 2. The basic reproductive number is given and dynamical behaviour of the system is analyzed in Section 3. Numerical simulations are given to demonstrate the main results in Section 4. Conclusion is finally drawn in Section 5.

\section{The SIRS Model with Discrete Delay}

Suppose that the size of the network is a constant $N$ during the period of epidemic spreading, we also suppose that the degree of each degree is time invariant. Let $S_{k}(t), I_{k}(t)$ and $R_{k}(t)$ be the relative density of susceptible nodes, infected nodes and recovered nodes of connectivity $k$ at time $t$, respectively. Obviously, the following normalization condition holds due to the fact that the number of total nodes with degree $k$ is a constant $p(k) N$ during the period of epidemic spreading.

$$
S_{k}(t)+I_{k}(t)+R_{k}(t)=1, k=1,2, \cdots, n .
$$

The dynamical equation for the density $S_{k}(t), I_{k}(t)$ and $R_{k}(t)$, at the mean-field level, satisfy the following system when $t>0$ :

$$
\left\{\begin{array}{l}
\dot{S}_{k}(t)=-\lambda(k) S_{k}(t) \Theta(t)+\delta \lambda(k) S_{k}(t-\tau) \Theta(t-\tau)+\mu R_{k}(t), \\
\dot{I}_{k}(t)=\lambda(k) S_{k}(t) \Theta(t)-\lambda(k) S_{k}(t-\tau) \Theta(t-\tau), \\
\dot{R}_{k}(t)=(1-\delta) \lambda(k) S_{k}(t-\tau) \Theta(t-\tau)-\mu R_{k}(t),
\end{array}\right.
$$

where $\lambda(k)$ is the correlated (k-dependent) infection rate such as $\lambda k$ [5] [8] [20], $\lambda c(k)$ [6] and so on, and $\tau$ represents the average infectious period. The term $\delta \lambda(k) S_{k}(t-\tau) \Theta(t-\tau)$ describes some infected nodes may become susceptible nodes because they are recovered and are not immunized, where $\delta$ is called incomplete cure rate, and consequently, $(1-\delta)$ is cure rate. In reality, for example, some computer users find network virus and kill it after $\tau$ due to its destruction of the user, but they do not take further action to immunize computer from the network virus, but the other users can get the technique to protect their computer and their computer cannot be infected again. The dynamics of $n$ groups of SIRS subsystems are coupled through the function $\Theta(t)$, which represents the probability that any given link points to an infected site. Assume that the network has no degree correlations [4], we have

$$
\Theta(t)=\frac{1}{\langle k\rangle} \sum_{k} \varphi(k) p(k) I_{k}(t),
$$

where $\langle k\rangle=\sum_{k} p(k) k$ stands for the average node degree, and $\varphi(k)$ has many different forms, such as $\varphi(k)=k$ in [4] [5], $\varphi(k)=A$ in [7], $\varphi(k)=k^{\alpha}, 0<\alpha<1$ in [8], and $\varphi(k)=a k^{\alpha} /\left(1+b k^{\alpha}\right), \quad 0<\alpha<1$ in [9] and so on.

The initial condition of system (1) is

$$
S_{k}(\theta)=\varphi_{k}(\theta), I_{k}(\theta)=\psi_{k}(\theta), R_{k}(\theta)=\phi_{k}(\theta), \theta \in[-\tau, 0],
$$

where $\omega=\left(\omega_{1}, \omega_{2}, \cdots, \omega_{n}\right) \in C$ are nonnegative continuous on $[-\tau, 0]$, and $\omega_{k}=\left(\varphi_{k}(\theta), \psi_{k}(\theta), \phi_{k}(\theta)\right)$ and 
$\varphi_{k}(0)>0, \psi_{k}(0)>0$ for $\theta=0 . C$ denote the Banach space $C\left([-\tau, 0], R^{3 n}\right)$ with the norm $\|\omega\|=\sup _{-h \leq \theta \leq 0}|\omega|$, and $|\omega|$ is Euclidean norm of $R^{3 n}$.

\section{Dynamical Behaviors of the Model}

Denote

$$
\begin{gathered}
S(t)=\left\langle S_{k}(t)\right\rangle, I(t)=\left\langle I_{k}(t)\right\rangle, R(t)=\left\langle R_{k}(t)\right\rangle, \\
R_{0}=\frac{\langle\lambda(k) \varphi(k)\rangle}{\langle k\rangle} \tau,
\end{gathered}
$$

where $\langle f(k)\rangle=\sum_{k=1}^{n} f(k) p(k)$ in which $f(k)$ is a function.

Obviously, the second equation of system (1) can be furthermore transformed into the following integral equation:

$$
I_{k}(t)=\lambda(k) \int_{t-\tau}^{t} S_{k}(s) \Theta(s) \mathrm{d} s
$$

Note that $S_{k}(t)+I_{k}(t)+R_{k}(t)=1$ and $R_{k}(t)$ can be replaced by $1-S_{k}(t)-I_{k}(t)$ in the first equation of system (1). Thus we obtain the following equivalent system of system (1):

$$
\left\{\begin{array}{l}
\dot{S}_{k}(t)=-\lambda(k) S_{k}(t) \Theta(t)+\delta \lambda(k) S_{k}(t-\tau) \Theta(t-\tau)+\mu\left(1-S_{k}(t)-I_{k}(t)\right), \\
I_{k}(t)=\lambda(k) \int_{t-\tau}^{t} S_{k}(s) \Theta(s) \mathrm{d} s .
\end{array}\right.
$$

Theorem 1. The system (5) has always a disease-free equilibrium $E_{0}(1,1, \cdots, 1,0,0, \cdots, 0)$. The system (5) has a unique endemic equilibrium $E_{*}\left(S_{1}^{*}, S_{2}^{*}, \cdots, S_{n}^{*}, I_{1}^{*}, I_{2}^{*}, \cdots, I_{n}^{*}\right)$ when $R_{0}>1$.

Proof. Denote $S_{k}=S_{k}^{*}, I_{k}=I_{k}^{*}$ and $R_{k}=R_{k}^{*}$ (some constants) and substitute them into (5), we have

$$
\left\{\begin{array}{l}
-\lambda(k) S_{k}^{*} \Theta^{*}+\delta \lambda(k) S_{k}^{*} \Theta^{*}+\mu\left(1-S_{k}^{*}-I_{k}^{*}\right)=0 \\
I_{k}^{*}=\lambda(k) S_{k}^{*} \Theta^{*} \tau
\end{array}\right.
$$

where

$$
\Theta^{*}=\langle k\rangle^{-1} \sum_{k}^{n} \varphi(k) p(k) I_{k}^{*}
$$

It yields that

$$
I_{k}^{*}=\frac{\lambda(k) \mu \Theta^{*} \tau}{((1-\delta)+\mu \tau) \lambda(k) \Theta^{*}+\mu} .
$$

Substituting it into (7), we obtain the self-consistency equality

$$
\Theta^{*}=\frac{1}{\langle k\rangle} \sum_{k}^{n} \varphi(k) p(k) \frac{\lambda(k) \mu \Theta^{*} \tau}{((1-\delta)+\mu \tau) \lambda(k) \Theta^{*}+\mu}=f\left(\Theta^{*}\right) .
$$

Obviously, $\Theta^{*}=0$ always satisfies (9), it follows that from (8) that the disease-free equilibrium $E_{0}$ of system (5) always exists. Note that

$$
\left.\frac{\mathrm{d} f\left(\Theta^{*}\right)}{\mathrm{d} \Theta^{*}}\right|_{\Theta^{*}=0}=\left.\frac{1}{\langle k\rangle} \sum_{k}^{n} \varphi(k) p(k) \frac{\lambda(k) \mu^{2} \tau}{\left(((1-\delta)+\mu \tau) \lambda(k) \Theta^{*}+\mu\right)^{2}}\right|_{\Theta^{*}=0}=\frac{\langle\lambda(k) \varphi(k)\rangle}{\langle k\rangle} \tau=R_{0}
$$

and 


$$
\frac{\mathrm{d} f^{2}\left(\Theta^{*}\right)}{\mathrm{d} \Theta^{* 2}}=\frac{1}{\langle k\rangle} \sum_{k}^{n} \varphi(k) p(k) \frac{\left.-2 \lambda(k) \mu^{2} \tau(((1-\delta)+\mu \tau) \lambda(k))\right)}{\left(((1-\delta)+\mu \tau) \lambda(k) \Theta^{*}+\mu\right)^{3}}<0
$$

Hence, if $R_{0}>1$, the Equation (9) has a unique positive solution, consequently, system (5) has a unique positive equilibrium $E_{*}\left(S_{1}^{*}, S_{2}^{*}, \cdots, S_{n}^{*}, I_{1}^{*}, I_{2}^{*}, \cdots, I_{n}^{*}\right)$ since (8) holds. $E_{*}$ is an unique endemic equilibrium.

Theorem 2. Consider the system (5), the following assertions hold.

(1) If $R_{0}<1$, the equilibrium $E_{0}$ of system (5) is globally attractive.

(2) If $R_{0}>1$, the disease is uniformly persistent, i.e., there exists a positive constant $\varepsilon$ such that $\lim _{t \rightarrow+\infty} \inf I(t)>\varepsilon$, and the equilibrium $E_{0}$ of system (5) is unstable.

Proof. First, According to the Equation (4), similar to the proof of Theorem 1 in [20], we can obtain that the equilibrium $E_{0}$ of system (5) is globally attractive.

Second, motivated by the work in [22], we will prove that conclusion (2) in Theorem 2 holds step by step, i.e., we prove that the disease is uniformly persistent when $R_{0}>1$.

Step 1. We will prove that for any $t_{0}>0$, it is impossible that $I_{k}(t)<\eta$ for $t>t_{0}$.

Since $R_{0}>1$, there exists a small enough $\eta>0$ such that

$$
R_{\eta}=\frac{\langle\lambda(k) \varphi(k) \Delta(k)\rangle}{\langle k\rangle} \tau>1
$$

in which $\Delta(k)=\frac{\mu(1-\eta)}{\lambda(k)\langle\varphi(k) \eta\rangle /\langle k\rangle+\mu}$.

Suppose $I_{k}(t)<\eta$ for $t>t_{0}$, which implies

$$
\Theta(t)<\frac{\langle\varphi(k) \eta\rangle}{\langle k\rangle}
$$

for $t>t_{0}$. It follows from the first equation of system (5) that

$$
\dot{S}_{k}(t) \geq \mu(1-\eta)-\left(\lambda(k) \frac{\langle\varphi(k) \eta\rangle}{\langle k\rangle}+\mu\right) S_{k}(t)
$$

for $t>t_{0}$. Hence there exists a $t_{1}>t_{0}$ such that

$$
S_{k}(t) \geq \frac{\mu(1-\eta)}{\lambda(k)\langle\varphi(k) \eta\rangle /\langle k\rangle+\mu}=\Delta(k)
$$

for $t>t_{1}$, and there a $t_{2}>t_{1}+\tau$ such that

$$
I_{k}(t)=\lambda(k) \int_{t-\tau}^{t} S_{k}(s) \Theta(s) \mathrm{d} s \geq \lambda(k) \Delta(k) \int_{t-\tau}^{t} \Theta(s) \mathrm{d} s
$$

It follows from (2) and (12) that

$$
\Theta(t) \geq R_{\eta} \frac{1}{\tau} \int_{t-\tau}^{t} \Theta(s) \mathrm{d} s
$$

Set $\Theta_{l}=\min _{t \in\left[t_{2}, t_{2}+\tau\right]} \Theta(t)$. We claim that $\Theta \geq \Theta_{l}$ for $t \geq t_{2}$. If not, there exists a $t_{3} \geq t_{2}+\tau$ such that $\Theta\left(t_{3}\right) \geq \Theta_{l}$ and $\Theta(t) \geq \Theta_{l}$ for $t_{2} \leq t \leq t_{3}$. It follow that

$$
\Theta\left(t_{3}\right) \geq R_{\eta} \frac{1}{\tau} \Theta_{l} \tau=R_{\eta} \Theta_{l}=R_{\eta} \Theta\left(t_{3}\right)
$$

which leads to $R_{\eta} \leq 1$, contradicting $R_{\eta}>1$. This proves the claim.

Choose a positive constant $R_{1}$ which satisfies $1<R_{1}<R_{\eta}$. We claim now that $\Theta(t)>R_{1} \Theta_{l}$ for all $t>t_{2}+\tau$. 
Note that

$$
\Theta\left(t_{2}+\tau\right) \geq R_{\eta} \frac{1}{\tau} \int_{t_{2}}^{t_{2}+\tau} \Theta(s) \mathrm{d} s \geq R_{\eta} \Theta_{l}>R_{1} \Theta_{l}
$$

If the claim is not valid, there exists a $t_{4}>t_{2}+\tau$ such that $\Theta\left(t_{4}\right)=R_{1} \Theta_{l}$ and $\Theta \geq R_{1} \Theta_{l}$ for $t_{2}+\tau \leq t \leq t_{4}$. Thus

$$
\Theta\left(t_{4}\right) \geq R_{\eta} \frac{1}{\tau} \int_{t_{4}-\tau}^{t_{4}} \Theta(s) \mathrm{d} s \geq R_{\eta} R_{1} \Theta_{l}=R_{\eta} \Theta_{l}=R_{\eta} \Theta\left(t_{4}\right)
$$

which leads to $R_{\eta} \leq 1$, contradicting $R_{\eta}>1$. This proves the claim. By induction method, we conclude that

$$
\Theta(t)>\Theta_{l} R_{1}^{n}
$$

for $t>t_{2}+n \tau$. It follows that $\Theta(t) \geq\langle\varphi(k) \eta\rangle /\langle k\rangle$ if $t$ is sufficiently large, contradicting $\Theta(t)<\langle\varphi(k) \eta\rangle /\langle k\rangle$. So, for any $t_{0}>0$, it is impossible that $I_{k}(t)<\eta$ for $t>t_{0}$.

Step 2. We will prove that there exists a positive constant $\varepsilon$ such that $\lim _{t \rightarrow+\infty} \inf I(t)>\varepsilon$.

Since it is impossible that $I_{k}(t)<\eta$ for $t>t_{0}$. Hence, there are two cases to be considered for $\Theta(t)$. Hence, there are two cases to be considered for $\Theta(t)$.

Case 1: $I_{k}(t) \geq \eta$ when $t$ is sufficiently large.

Case 2: $I_{k}(t)$ is oscillates about $\eta$ when $t$ is sufficiently large.

Suppose $I_{k}\left(t^{*}\right)=I_{k}\left(t^{*}+q\right)=\eta$ and $I_{k}(t) \leq \eta$ for $t^{*} \leq t \leq t^{*}+q$, where $t^{*}>t_{1}+\tau$ is sufficiently large such that $I_{k}(t)<\eta$ holds. Consequently, $\Theta\left(t^{*}\right)=\Theta\left(t^{*}+q\right)=\langle\varphi(k) \eta\rangle /\langle k\rangle$ and $\Theta(t) \leq\langle\varphi(k) \eta\rangle /\langle k\rangle$ for $t^{*} \leq t \leq t^{*}+q \cdot \Theta(t)$ is uniformly continuous since the positive solutions of system (4) are bounded. Hence, there is a $0<\sigma<\tau$ (independent of the choice of $t^{*}$ ) such that $\Theta(t)>\langle\varphi(k) \eta\rangle /(2\langle k\rangle)$ for $t^{*} \leq t \leq t^{*}+\sigma$. If $q<\sigma$, there is nothing to prove. If $\sigma<q \leq \tau$, for $t^{*}+\sigma<t<t^{*}+q$, we have from (15) that

$$
\Theta(t) \geq R_{\eta} \frac{1}{\tau} \int_{t-\tau}^{t} \Theta(s) \mathrm{d} s>R_{\eta} \frac{1}{\tau} \int_{t^{*}}^{t^{*}+\sigma} \frac{\langle\varphi(k) \eta\rangle}{2\langle k\rangle} \mathrm{d} s=R_{\eta} \frac{\langle\varphi(k) \eta\rangle \sigma}{2\langle k\rangle} \tau .
$$

Let us define $p=\min \left\{R_{\eta}\langle\varphi(k) \eta\rangle \sigma /(2\langle k\rangle \tau),\langle\varphi(k) \eta\rangle /(2\langle k\rangle)\right\}$, then $\Theta(t) \geq p$ for $t^{*}<t<t^{*}+q$. If $q<\tau$, by similar method in step 1 , we can obtain that $\Theta(t) \geq p$ for $t^{*}<t<t^{*}+q$. Thus, for case 2, $\Theta(t) \geq p$ when $t$ is sufficiently large, and $I_{k}(t) \geq \varepsilon_{1}$ where $\varepsilon_{1}=\langle k\rangle p / \min _{\{k=1,2, \cdots, n\}} \varphi(k)$.

Hence, $I_{k}(t) \geq \varepsilon$ when $t$ is sufficiently large, where $\varepsilon=\min \left\{\eta, \varepsilon_{1}\right\}$, consequently, $\lim _{t \rightarrow+\infty} I(t) \geq \sum_{k=1}^{n} p(k) \varepsilon=\varepsilon$, and the disease is uniformly persistent.

At last, since $\lim _{t \rightarrow+\infty} I_{k}(t) \geq \varepsilon$ when $R_{0}>1$, the equilibrium $E_{0}$ is unstable when $R_{0}>1$. This completes the proof of Theorem 2 .

The basic reproductive number for system (4) is $R_{0}=\tau\langle\lambda(k) \varphi(k)\rangle /\langle k\rangle$. If $R_{0}<1$, the disease will disappear due to the global attractivity of $E_{0}$. If $R_{0}>1$, the disease will always exists due to $\lim _{t \rightarrow+\infty} \inf I(t) \geq \varepsilon>0$.

Remark. When $\delta=1$ system (1) reduce to system (4) in [20], and the results still holds.

\section{Numerical Simulations}

The basic reproductive number for system (1) is

$$
R_{0}=\frac{\langle\lambda(k) \varphi(k)\rangle}{\langle k\rangle} \tau \text {. }
$$


Note that an epidemic always occurs on a finite networks in the real world, the maximum connectivity $n$ of any node is related to the network age, which is measured as the number of nodes $N$ [8] [9]:

$$
n=m N^{1 /(\gamma-1)} \text {, }
$$

where $m$ is the minimum connectivity of the network. It follows from (14) and (15) that $R_{0}$, which depends both $N$ and $\tau$, can be approximately computed.

Now we present the results of numerical simulations by using MATLAB 7.0 to support the results obtained in previous sections. Since the equilibria were obtained from system (5), the simulations are based on system (5) and a scale-free network in which the degree distribution is $p(k)=C k^{-\gamma}$, and $C$ satisfies $\sum_{k=1}^{n} p(k)=1$. Assume the network is a finite network with $n=100$ and $m=1$ are suitable assumptions. Let $\gamma=0.5$, $\mu=0.3, \delta=0.2, \tau=2, \phi(k)=a k^{\alpha} /\left(1+b k^{\alpha}\right)$ in which $a=0.5, \alpha=0.75, b=0.02$, and $\lambda(k)=\lambda k$. Figures 1-3 show the dynamic behaviors of system (5).

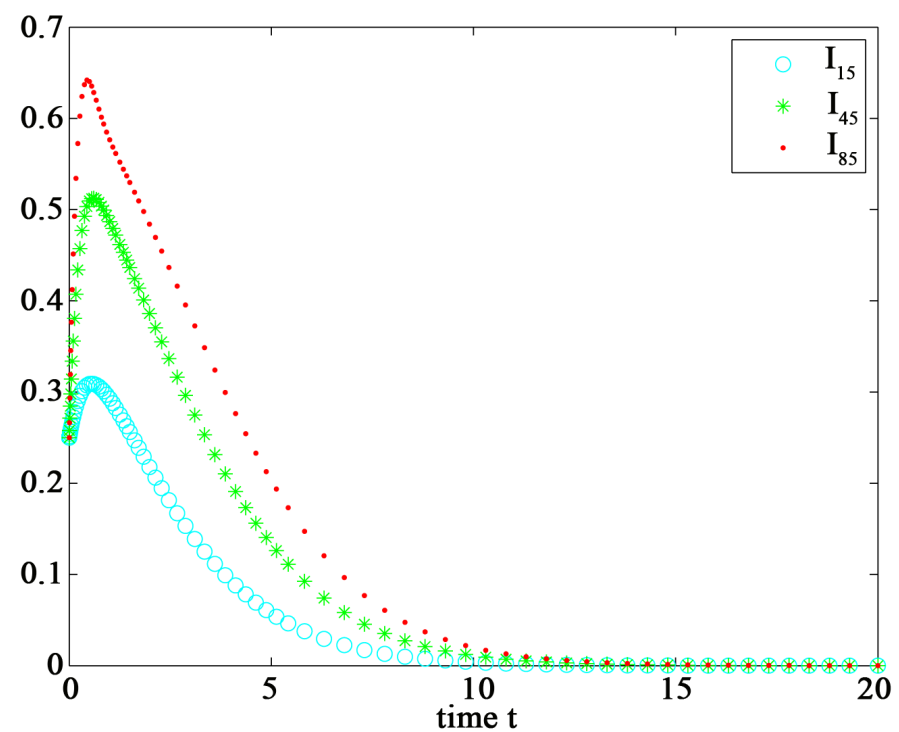

Figure 1. The time series of system (5) with $\lambda(k)=0.5 k$ and $R_{0}=0.9644<1$.

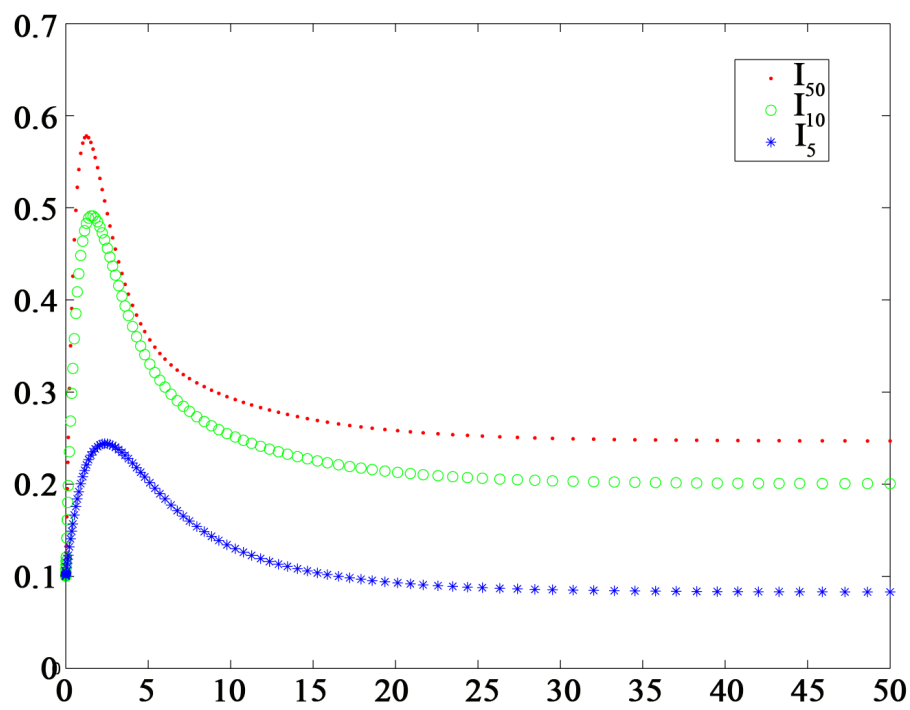

Figure 2. The time series of system (5) with $\lambda(k)=0.5 k$ and $R_{0}=1.9288>1$. 


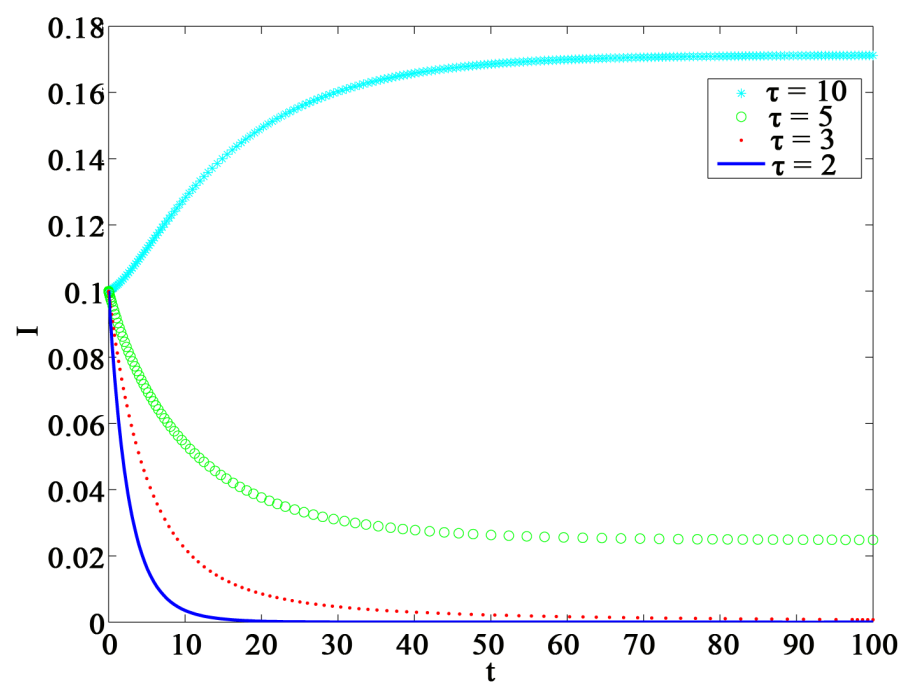

Figure 3. The time series of system (5) with $\lambda(k)=0.5 k$ and $\pi=2,3,5,10$.

From the dynamical behaviors of the SIRS model (5) shown in Figure I and Figure 2, it can be seen that if $R_{0}<1$, the infection eventually disappears. If $R_{0}>1$, the relative density of infected nodes will tend to a positive constant, and the infection will always exist. The numerical results are consistent with the theoretical results.

According to $R_{0}$, if $R_{0}<1$, the equilibrium $E_{0}$ is globally attractive and the disease eventually disappear. However, $R_{0}>1$ may hold as $\tau$ increases due to the fact $R_{0}$ is proportional to $\tau$, that is to say, the equilibrium $E_{0}$ may lose its stability when the average infection period of disease $\tau$ is large enough and the infection will always exist (shown in Figure 3).

\section{Conclusion}

An SIRS model with discrete delay has been proposed for investigating the dynamical behaviors of the epidemics on scale-free networks. Through mathematical analysis, we obtained the basic reproduction number $R_{0}$. The main results reveal that when $R_{0}<1$, the disease-free equilibrium is globally attractive, while $R_{0}>1$, the disease-free equilibrium is unstable and the disease is uniformly persistent. In addition, numerical simulations show that the endemic equilibrium $E_{*}$ is globally asymptotically stable when $R_{0}>1$ (as shown in Figure 2). We would like to mention here that it is interesting but challenging to discuss the stability of equilibrium $E_{*}$, we leave this for our future work.

\section{References}

[1] Barabási, A.L. and Alber, R. (1999) Emergence of Scaling in Random Networks. Science, 286, 509-512. http://dx.doi.org/10.1126/science.286.5439.509

[2] Small, M. and Tse, C.K. (2005) Small World and Scale Free Model of Transmission of SARS. International Journal of Bifurcation and Chaos, 15, 1745-1755. http://dx.doi.org/10.1142/S0218127405012776

[3] Small, M., Walker, D.M. and Tse, C.K. (2007) Scale Free Distribution of Avian Influenza Outbreaks. Physical Review Letters, 99, Article ID: 188702. http://dx.doi.org/10.1103/PhysRevLett.99.188702

[4] Pastor-Satorras, R. and Vespignani, A. (2001) Epidemic Spreading in Scale Free Networks. Physical Review Letters, 86, 3200-3203. http://dx.doi.org/10.1103/PhysRevLett.86.3200

[5] Pastor-Satorras, R. and Vespignani, A. (2002) Epidemic Dynamics in Finite Size Scale-Free Networks. Physical Review E, 65, Article ID: 035108. http://dx.doi.org/10.1103/PhysRevE.65.035108

[6] Olinky, R. and Stone, L. (2004) Unexpected Epidemic Thresholds in Heterogeneous Networks: The Role of Disease Transmission. Physical Review E, 70, Article ID: 030902. http://dx.doi.org/10.1103/PhysRevE.70.030902

[7] Yang, R., Ren, J., et al. (2007) Epidemic Spreading on Hererogeneous Networks with Identical Infectivity. Physics Letters A, 364, 189-193. http://dx.doi.org/10.1016/j.physleta.2006.12.021

[8] Cheng, X., Liu, X., Chen, Z. and Yuan, Z. (2009) Spreading Behavior of SIS Model with Non-Uniform Transmission 
on Scale-Free Networks. The Journal of Universities of Posts and Telecommunications, 16, 27-31. http://dx.doi.org/10.1016/S1005-8885(08)60173-9

[9] Zhang, H. and Fu, X. (2009) Spreading of Epidemics on Scale-Free Networks with Nonlinear Infectivity. Nonlinear Analysis, 70, 3273-3278. http://dx.doi.org/10.1016/j.na.2008.04.031

[10] Li, K., Small, M., Zhang, H. and Fu, X. (2010) Epidemic Outbreaks on Networks with Effective Contacts. Nonlinear Analysis: RWA, 11, 1017-1025. http://dx.doi.org/10.1016/j.nonrwa.2009.01.046

[11] Fu, X.C., Small, M., Walker, D.M. and Zhang, H.F. (2008) Epidemic Dynamics on Scale-Free Networks with Piecewise Linear Infectivity and Immunization. Physical Review E, 77, Article ID: 036113. http://dx.doi.org/10.1103/PhysRevE.77.036113

[12] Zhang, J.P. and Jin, Z. (2011) The Analysis of an Epidemic Model on Networks. Applied Mathematics and Computation, 217, 7053-7064. http://dx.doi.org/10.1016/j.amc.2010.09.063

[13] Zhu, G.H., Fu, X.C. and Chen, G.R. (2013) Global Attractivity of a Network-Based Epidemics SIS Model with Nonlinearn Infectivity. Communications in Nonlinear Science and Numerical Simulation, 17, 2588-2594.

[14] Gong, Y.W., Song, Y.R. and Jiang, G.P. (2012) Epidemic Spreading in Scale-Free Networks Including the Effect of Individual Vigilance. Chinese Physics B, 21, Article ID: 010205. http://dx.doi.org/10.1088/1674-1056/21/1/010205

[15] Li, T., Wang, Y.M. and Guan, Z.H. (2014) Spreading Dynamics of a SIQRS Epidemic Model on Scale-Free Networks. Communications in Nonlinear Science and Numerical Simulation, 19, 686-692. http://dx.doi.org/10.1016/j.cnsns.2013.07.010

[16] Liu, J.L. and Zhang, T.L. (2011) Epidemic Spreading of an SEIRS Model in Scale-Free Networks. Communications in Nonlinear Science and Numerical Simulation, 16, 3375-3384. http://dx.doi.org/10.1016/j.cnsns.2010.11.019

[17] Chen, L.J. and Sun, J.T. (2014) Global Stability and Optimal Control of an SIRS Epidemic Model on Heterogeneous Networks. Physica A: Statistical Mechanics and Its Applications, 410, 196-204. http://dx.doi.org/10.1016/j.physa.2014.05.034

[18] Ma, Z.E. and Li, J. (2009) Dynamical Modelling and Analysis of Epidemics. World Scientific Publishing Company, Singapore.

[19] Liu, X. and Xu, D.J. (2012) Analysis of $S E^{\tau} I R^{\omega} S$ Epidemic Disease Models with Vertical Transmission in Complex Networks. Acta Mathematicae Applicatae Sinica, English Series, 28, 63-74. http://dx.doi.org/10.1007/s10255-012-0094-1

[20] Liu, Q.M., Deng, C.S. and Sun, M.C. (2014) The Analysis of an Epidemic Model with Time Delay on Scale-Free Networks. Physica A: Statistical Mechanics and Its Applications, 410, 79-87. http://dx.doi.org/10.1016/j.physa.2014.05.010

[21] Wang, J.R., Wang, J.P., Liu, M.X. and Liu, Y.W. (2014) The Analysis of an Epidemic Model with Time Delay on Scale-Free Networks. Physica A: Statistical Mechanics and Its Applications, 410, 268-275. http://dx.doi.org/10.1016/j.physa.2014.05.011

[22] Wang, W.D. and Ma, Z.E. (2002) Global Dynamics of an Epidemic Model with Time Delay. Nonlinear Analysis: Real World Applications, 3, 365-373. http://dx.doi.org/10.1016/S1468-1218(01)00035-9 